Proceedings of the Edinburgh Mathematical Society (2006) 49, 567-573 (C)

DOI:10.1017/S0013091504001099 Printed in the United Kingdom

\title{
MÜLLER'S QUESTION ON SEMI-PERFECT COMPLETE HEREDITARY NOETHERIAN PRIME RINGS
}

\author{
PHẠM NGỌC ÁNH ${ }^{1}$ AND DOLORS HERBERA ${ }^{2}$ \\ ${ }^{1}$ Mathematical Institute, Hungarian Academy of Sciences, \\ PO Box 127, 1364 Budapest, Hungary (anh@renyi.hu) \\ ${ }^{2}$ Departament de Matemàtiques, Universitat Autònoma de Barcelona, \\ 08193 Bellaterra, Barcelona, Spain (dolors@manwe.mat.uab.es)
}

(Received 21 September 2004)

\begin{abstract}
A positive answer to a question of Müller is given: any semi-perfect complete hereditary Noetherian prime ring $R$ has a weakly symmetric self-duality sending every ideal $I$ to its cycleneighbour $X$. Consequently, the factor rings $R / I$ and $R / X$ are isomorphic without using the 1984 results of Dischinger and Müller.
\end{abstract}

Keywords: Morita duality; Noetherian prime rings; hereditary; injective modules; localizations; quotient rings

2000 Mathematics subject classification: Primary 16D50; 16D90; 16E60

\section{Introduction}

Let ${ }_{R} U_{R}$ be a bimodule. For any subsets $X \subseteq R, V \subseteq U$ let

$$
X^{*}=\{u \in U \mid X u=0\}, \quad V^{*}=\{r \in R \mid V r=0\} .
$$

The map $I \mapsto I^{* *}, I \triangleleft R$ is an endomorphism of the ideal lattice of $R$. Motivated by the corresponding notions for hereditary Noetherian prime (HNP) rings, this map is called a cycle map and the orbits of maximal ideals of $R$ are said to be cycles with respect to a bimodule $U$. Since throughout the paper it is always clear which bimodule induces the considered cycle map, we denote by $I^{* *}$ the cycle-neighbour of an ideal $I$, omitting the indication of the corresponding bimodule.

If $U=Q / R$, where $Q$ is the classical quotient ring of a semi-perfect (in the radial topology) complete HNP ring $R$ (assumed always to be a non-Artinian, basic, non-local ring), then $U$ induces a self-duality for $R$. Inspired by the fact that proper factors of HNP rings are finite direct sums of indecomposable Artinian serial rings, and the latter have weakly symmetric self-duality (cf. $[\mathbf{3}])$, in $[\mathbf{9}, \mathbf{1 0}]$ Müller asks whether there exists an automorphism $\alpha$ of $R$ such that it sends a non-zero ideal $I$ onto its cycle-neighbour $I^{* *}$. We will answer Müller's question in the positive without using the result in [3]. 
If $S$ is any non-zero, localizable semi-prime ideal of $R$, then it is well known (cf. [11]) that both the localization $R_{S}$ and its completion with respect to the radical topology are semi-local HNP rings. This shows that the mild condition of completeness is in fact a technical one that makes the essence clear and simplifies the situation.

\section{Preliminaries}

We recall the close relation between automorphisms and dualities described in [8]. Since the results are consequences of long theorems in [8], for the sake of completeness and self-containedness we insert a short proof of them. More detailed discussion can be found in $[6]$.

A Morita duality (henceforth called a duality for brevity) between two rings $R$ and $T$ is a contravariant equivalence between certain full subcategories of left $R$-modules and right $T$-modules respectively, both of which contain all the finitely generated modules and are both closed under taking submodules and factor modules. Such a duality is called a selfduality if $R$ and $T$ are isomorphic rings. A duality is representable, i.e. it is equivalent to functors $\operatorname{Hom}_{R}(-, U)$ and $\operatorname{Hom}_{T}(-, U)$ for some appropriate bimodule ${ }_{R} U_{T}$. If ${ }_{R} U_{T}$ and ${ }_{R} V_{T}$ induce two dualities between $R$ and $T$, then these dualities are naturally equivalent if and only if ${ }_{R} U_{T}$ and ${ }_{R} V_{T}$ are isomorphic as bimodules. A bimodule ${ }_{R} U_{T}$ is called faithfully balanced if $R=\operatorname{End}\left(U_{T}\right)$ and $T=\operatorname{End}\left({ }_{R} U\right)$.

We denote by $\operatorname{Aut}(R)$ and $\operatorname{Aut}(T)$ the groups of ring automorphisms of $R$, and $T$, respectively. For a bimodule ${ }_{R} U_{T}$ and each couple of automorphisms $\alpha \in \operatorname{Aut}(R), \beta \in$ $\operatorname{Aut}(T)$ we define a new bimodule structure on $U$ by putting

$$
r * u * t=\alpha(r) u \beta(t) \quad \forall r \in R, u \in U, t \in T .
$$

Following the notation in [2], this new bimodule is denoted by ${ }_{\alpha} U_{\beta}$. For example, ${ }_{1} U_{1}=$ $U$.

The proof of the following statement is similar to that of [2, Proposition II.5.2] or [4, Lemma III.12.16].

Proposition 2.1. Let $R$ and $T$ be rings, and let ${ }_{R} U_{T}$ and ${ }_{R} V_{T}$ be faithfully balanced bimodules. If $f:{ }_{R} U \rightarrow{ }_{R} V$ is a left $R$-module isomorphism, then there is an automorphism $\beta \in \operatorname{Aut}(T)$ such that

(i) $f$ becomes an isomorphism between the bimodules ${ }_{1} U_{\beta}$ and ${ }_{1} V_{1}$;

(ii) $\beta$ is an inner automorphism if and only if the bimodules ${ }_{1} U_{1}$ and ${ }_{1} V_{1}$ are isomorphic.

Moreover, if $g:{ }_{R} U \rightarrow{ }_{R} V$ is another left $R$-module isomorphism, and it induces an automorphism $\beta^{\prime}$, then there exists $x \in T$ such that $\beta=x^{-1} \beta^{\prime} x$.

Proof. For each element $t \in T$, the map

$$
\beta_{t}:{ }_{R} U \rightarrow{ }_{R} U: u \in U \mapsto((u) f t) f^{-1}
$$


is an $R$-endomorphism of ${ }_{R} U$; hence, it is an element of $T$. It is routine to check that the map $\beta: T \rightarrow T: t \mapsto \beta(t)=\beta_{t}$ is a ring automorphism that makes ${ }_{1} U_{\beta}$ isomorphic to $V$ in view of

$$
(r u * t) f=\left((r u) \beta_{t}\right) f=\left\{[(r u) f t] f^{-1}\right\} f=(r u) f t=r((u) f) t \quad \text { for all } r \in R, t \in T .
$$

Assume that $h:{ }_{1} V_{1} \rightarrow{ }_{1} U_{1}$ is a bimodule isomorphism. Then $y=f h \in T$ has the inverse $x \in T$. By definition, we have, for each $u \in U, t \in T$,

$$
\begin{aligned}
(u) \beta(t)=(u) \beta_{t} & =[(u) f t] f^{-1}=\left[(u) y h^{-1} t\right] h y^{-1}=\left\{\left[\left((u) y h^{-1}\right) t\right] h\right\} y^{-1} \\
& =\left\{\left[(u) y h^{-1} h\right] t\right\} y^{-1}=(u)\left(y t y^{-1}\right)=(u)\left(x^{-1} t x\right) .
\end{aligned}
$$

Thus, $\beta$ is an inner automorphism induced by $x$. Conversely, if $\beta$ is an inner automorphism induced by a unit $x \in T$, then, for all elements $t \in T, u \in U$,

$$
(u t)(f x)=\left(u \beta\left(x t x^{-1}\right)\right)(f x)=\left((u) \beta_{x t x^{-1}}\right)(f x)=\left[\left((u) f x t x^{-1}\right) f^{-1}\right](f x)=(u)[(f x) t] .
$$

Consequently, $f x$ is a bimodule isomorphism between $U$ and $V$.

The last statement can be checked directly by taking $x=g f^{-1}$.

The outer automorphism class group $\operatorname{Out}(T)$ of $T$ is the factor group of $\operatorname{Aut}(T)$ by the normal subgroup of all inner automorphisms. Proposition 2.1 says exactly that, for a faithfully balanced bimodule ${ }_{R} U_{T}$, there is a bijective correspondence between the bimodule structures on the left $R$-module ${ }_{R} U$ and the elements of $\operatorname{Out}(T)$. Moreover, if $f$ is a left $R$-module isomorphism between faithfully balanced bimodules ${ }_{R} U_{T},{ }_{R} V_{T}$ with the associated automorphism $\beta$ making $f$ a bimodule isomorphism between ${ }_{1} U_{\beta}$ and $V$, then $f$ is clearly a right $T$-module isomorphism between $U_{T}$ and $V_{\beta^{-1}}$. Consequently, again by Proposition 2.1 there is a ring automorphism $\alpha$ of $R$ such that $f$ is a bimodule isomorphism between bimodules ${ }_{\alpha} U_{1}$ and ${ }_{1} V_{\beta^{-1}}$. However, it is not clear whether there is a natural correspondence between automorphisms $\alpha$ of $R$ and isomorphisms $f$ of the left $R$-module ${ }_{R} U$. If $U$ is an essential extension of the direct sum $S$ of all pairwise nonisomorphic simple modules such that $S$ is of finite length and any automorphism of $S$ can be extended to $U$, then it is clear that the groups $\operatorname{Out}(R)$ and $\operatorname{Out}(T)$ are isomorphic. In particular, if $\left(R,{ }_{R} U_{T}, T\right)$ is a duality where $R$ and $T$ are basic rings, then the socle of $U$ is isomorphic to the direct sum of all pairwise non-isomorphic simple modules. Therefore, the groups $\operatorname{Out}(R)$ and $\operatorname{Out}(T)$ are isomorphic and they characterize all the equivalence classes of dualities between $R$ and $T$. In the general case, choose $e^{2}=e \in R$, $f^{2}=f \in T$ such that $R$ and $T$ are Morita equivalent to the basic rings $A=e R e$ and $B=f T f$, respectively. Then $e U_{T}$ and ${ }_{R} U f$ are the minimal injective co-generators for $R$ and $T$, respectively, i.e. their socles are the direct sum of all pairwise non-isomorphic simple modules. Moreover, $\left(A,{ }_{A} e U f_{B}, B\right)$ is also a duality. Thus, the groups $\operatorname{Out}(A)$ and $\operatorname{Out}(B)$ are isomorphic. More generally, let $\left(R,{ }_{R} U_{T}, T\right)$ be a Baer duality, i.e. the lattices of submodules of ${ }_{R} R$ and $U_{T}$ as well as ${ }_{R} U$ and $T_{T}$, respectively, are anti-isomorphic. (For the foundation of Baer duality we refer the reader to [1].) By [1, Corollary 3.4], any automorphism of the socle of ${ }_{R} U$ or $U_{T}$ can be extended to an automorphism of ${ }_{R} U$ 
and $U_{T}$, respectively. Therefore, if $U$ is in addition faithfully balanced, then, in view of [1, Proposition 2.5], one can obtain a slight generalization of [8, Theorem 8.3] as follows.

Theorem 2.2. If $\left(R,{ }_{R} U_{T}, T\right)$ is a Baer duality such that $U$ is faithfully balanced or, in particular, a Morita duality, then $\operatorname{Out}(A)$ and $\operatorname{Out}(B)$ are isomorphic, where $A$ and $B$ are the associated basic rings of $R$ and $T$, respectively.

Remark 2.3. ${ }_{R} U$ and $\bar{\alpha}_{\bar{\alpha}} U$ are in general not isomorphic modules over $R$. Let $F$ be any field and $R$ be a direct sum of two copies of $F$, denoted by $A$ and $B$. Then ${ }_{R} A,{ }_{R} B$ are all types of simple left $R$-modules. Let $W$ be the direct sum of two copies of ${ }_{R} B$. Put $U={ }_{R} A \oplus W, T=\operatorname{End}(U)$ and $C=\operatorname{End}(W)$. Then $T$ is a direct sum of the rings $A$ and $C$. Let $\alpha$ be the automorphism of $R$ interchanging $A$ and $B$. It is then easy to check that ${ }_{\alpha} U \cong{ }_{R} A^{2} \oplus{ }_{R} B$ and, hence, that ${ }_{R} U$ and ${ }_{\alpha} U$ are non-isomorphic left modules over $R$.

\section{A solution to a problem of Müller}

The most intriguing puzzle in the duality theory is the question of self-duality. No reasonable condition is known to determine whether a ring with duality also has a self-duality, and, since there are no specific conditions on the nature of the isomorphism between $R$ and $T=\operatorname{End}\left({ }_{R} U\right)$, even in the case when a ring is known to have self-duality it is difficult to determine whether it has a self-duality satisfying certain 'nice' properties.

If a bimodule ${ }_{R} U_{R}$ gives a self-duality for $R$, and $I$ is a two-sided ideal of $R$, then $I^{*}$ induces a duality between $R / I$ and $R / I^{* *}$. Therefore, we say that a duality induced by $U$ is a good duality if $I=I^{* *}$ for each ideal $I$ of $R$ or, equivalently, the cycle map induced by $U$ is the identity map. A weaker notion of good duality is one of weakly symmetric self-duality. A bimodule ${ }_{R} U_{R}$ induces a weakly symmetric self-duality for $R$ if $\operatorname{Hom}_{R}\left(R e / J(R) e,{ }_{R} U\right) \cong e R / e J(R)$ for every primitive idempotent $e \in R$. Therefore, a quasi-Frobenius ring is weakly symmetric if and only if the Nakayama permutation on the set of basic pairwise orthogonal primitive idempotents is the identity. In the following obvious proposition we explain this concept.

Proposition 3.1. For a self-dual ring $R$ with respect to a bimodule ${ }_{R} U_{R}$, the following statements are equivalent:

(i) $U$ gives a weakly symmetric self-duality;

(ii) $\operatorname{Soc}(U)$ and $R / J(R)$ are isomorphic as $R-R$-bimodules;

(iii) $M=M^{* *}$ for each maximal two-sided ideal $M$ of $R$.

All self-dualities of local rings are weakly symmetric. As observed in [10], a natural example of self-duality that is not weakly symmetric is given by non-local complete HNP rings. We shall briefly describe this example. 
In view of [7, Corollary 6.5], if $R$ is a basic non-local complete HNP ring, then there exists $n \geqslant 1$, and a complete rank-one valuation domain $F$, with maximal ideal $M=$ $F a=a F$ and classical quotient division $\operatorname{ring} K$, such that

$$
R=\left\{\left(a_{i j}\right) \in M_{n}(F) \mid a_{i j} \in M, \forall i>j\right\} .
$$

The Jacobson radical of $R$ is

$$
J(R)=\left\{\left(a_{i j}\right) \in M_{n}(F) \mid a_{i j} \in M, \forall i \geqslant j\right\},
$$

and the classical quotient of $R$ is the matrix $\operatorname{ring} M_{n}(K)=Q$. Put $U=Q / R$. For $k=1, \ldots, n$, let $e_{k}=\left(a_{i j}\right) \in R$ be a matrix which has only one non-zero entry, $a_{k k}=1$. Then $\left\{e_{1}, \ldots, e_{n}\right\}$ is a complete set of pairwise orthogonal primitive idempotents of $R$ and $1=e_{1}+\cdots+e_{n}$.

Routine calculations show that $e_{i} R / e_{i} J(R)$ is the socle of $e_{i+1} U$ for any $i=1, \ldots, n-1$, and that $e_{n} R / e_{n} J(R)$ is the socle of $e_{1} U$.

Therefore, for $n>1$, the self-duality of $R$ induced by $U$ is never weakly symmetric and we recognize through duality theory the cyclic quiver $\tilde{A}_{n}, n>1$. Note that, with a few obvious exceptions, all proper factor rings of $R$ are basic, connected Artinian serial rings whose associated quiver is $\tilde{A}_{n-1}$.

Since a simplified version of [5, Proposition 4.1] plays a key role in our solution to Müller's problem, we reproduce it, together with a detailed proof.

Proposition 3.2. Every weakly symmetric self-duality of an Artinian serial ring is a good duality.

Proof. Let $R$ be an Artinian serial ring and ${ }_{R} U_{R}$ be a bimodule such that $U$ induces a weakly symmetric self-duality for $R$. We have to show $I=I^{* *}$ for every ideal $I$ of $R$. Put $X=I^{* *}$ and let $1=e_{1}+\cdots+e_{n}$ be a decomposition of 1 as a sum of pairwise orthogonal primitive idempotents $e_{i}$. For all $i$, let $R_{i}=e_{i} R e_{i}, U_{i}=e_{i} U e_{i}$. To complete the proof it is sufficient to see that $e_{i} I e_{R_{R_{j}}}$ and $e_{i} X e_{j_{R_{j}}}$ have the same length for all $i, j$ because the $R_{i} e_{i} R e_{R_{j}}$ are all uniserial both as a left and a right monoid. Since $U$ induces a weakly symmetric self-duality, $U_{i}$ induces a self-duality for $R_{i}$ which sends $e_{i} R$ to $U e_{i}$. Consequently, by putting $V_{i}=U e_{i}, W_{i}=I^{*} e_{i}$ for all $i$, we have

$$
\begin{aligned}
\lg \left(e_{i} X e_{j_{R_{j}}}\right) & =\lg \left(R_{j} e_{j}\left(V_{i} / W_{i}\right)\right)=\lg \left(R_{j} e_{j} V_{i}\right)-\lg \left(R_{j} e_{j} W_{i}\right) \\
& =\lg \left(e_{i} R e_{j_{j}}\right)-\lg \left(e_{i}(R / I) e_{j_{R_{j}}}\right)=\lg \left(e_{i} I e_{j_{R_{j}}}\right),
\end{aligned}
$$

from which the statement follows. Here 'lg' denotes the length of modules.

A complete answer to Müller's question is given in the following theorem.

Theorem 3.3. Let $R$ be a semi-perfect complete HNP ring with the semi-simple Artinian classical quotient ring $Q$ and $U=Q / R$.

(i) If $\alpha$ is a ring automorphism of $R$ such that ${ }_{1} U_{\alpha}$ induces a weakly symmetric selfduality, then ${ }_{1} U_{\alpha}$ is a good duality. In particular, any weakly symmetric self-duality of $R$ is a good duality. 
(ii) There is an automorphism $\alpha$ of $R$ such that ${ }_{1} U_{\alpha}$ induces a weakly symmetric selfduality.

Proof. Since $R / Z$ is Artinian for any non-zero ideal $Z$, we have $J(R)^{n} \subset Z$ for some $n$.

(i) Let $\alpha$ be an automorphism of $R$ such that ${ }_{1} U_{\alpha}$ induces a weakly symmetric selfduality for $R$. We have to show that $\alpha(I)=I^{* *}$ for any ideal $I$ of $R$. Put $X=I^{* *}$. Since every automorphism fixes the Jacobson radical, we have $\alpha\left(J(R)^{n}\right)=J(R)^{n}$ for any natural number $n$.

Without loss of generality we can assume that $I$ is a non-zero proper ideal of $R$. Hence, $X$ is also non-zero. As $R$ is prime, $I \cap X \neq 0$. Therefore, there is a natural number $n$ with $J(R)^{n} \subset I \cap X$. Let $V=\left(J(R)^{n}\right)^{*}$. Then $V$ induces a self-duality for $\bar{R}=R / J(R)^{n}$, where the overbar, such that $R \rightarrow R / J(R)^{n}$, denotes the canonical projection. It is clear that $\bar{X}=(\bar{I})^{* *}$ holds where the considered cycle map is induced by the bimodule $V$ over $\bar{R}$. The equality $\alpha\left(J(R)^{n}\right)=J(R)^{n}$ implies that $\alpha$ induces, in the natural way, an automorphism $\bar{\alpha}$ of $\bar{R}$. Since, by assumption, ${ }_{1} U_{\alpha}$ is a weakly symmetric self-duality of $R$, ${ }_{1} V_{\bar{\alpha}}$ is also a weakly symmetric self-duality of the Artinian serial ring $\bar{R}$. By Proposition $3.2, \bar{\alpha}$ is a good duality. Hence,

$$
\bar{\alpha}(\bar{I})=\bar{X}, \quad \text { consequently } \alpha(I)=X .
$$

This completes the proof of the fact that $\alpha$ is a good duality.

(ii) To show the existence of an automorphism $\alpha$ of $R$ that makes ${ }_{1} U_{\alpha}$ a weakly symmetric self-duality for $R$, let $W=Q / J(R)$. It is well known (and easy to check) that $W$ is an injective envelope of $R / J(R)$ both as a left and as a right $R$-module. Since $R$ has a self-duality induced by $U, W$ also induces a self-duality which, in addition, it is weakly symmetric. Since ${ }_{R} U$ and ${ }_{R} W$ are injective envelopes of $R / J(R)$, they are isomorphic left modules. Let $f:{ }_{R} U \rightarrow{ }_{R} W$ be any isomorphism. In view of Proposition 2.2, there is an automorphism $\alpha$ of $R$ such that $f$ is an $R-R$-bimodule isomorphism between ${ }_{1} U_{\alpha}$ and $W$. This completes our proof.

We do not know whether a self-dual ring admits a good duality if all of its factor rings are self-dual. This question can be formulated in the following more general setting. Let $U$ be a faithfully balanced $R-R$-bimodule such that the factor rings $R / I, R / I^{* *}$ are isomorphic. Does there exist an automorphism $\alpha$ of $R$ sending every ideal $I$ of $R$ to its ideal-neighbour $I^{* *}$ with respect to the cycle map induced by $U$ ?

Acknowledgements. P.N.A. was partly supported by the Hungarian National Foundation for Scientific Research, grant no. T034530. Part of this paper was written while P.N.A. was visiting the Centre de Recerca Matemàtica supported by a grant from the Ministerio de Educación y Ciencia (Spain).

D.H. was supported by the DGESIC (Spain) through the project PB98-0873, by the Comissionat per Universitats i Recerca de la Generalitat de Catalunya and partly by the Hungarian National Foundation for Scientific Research, grant no. T043034. 


\section{References}

1. P. N. Ánh, D. Herbera and C. Menini, Baer and Morita duality, J. Alg. 232 (2000), 462-484.

2. H. BAss, Algebraic K-theory (Benjamin, New York, 1968).

3. F. Dischinger AND W. MülLER, Einreihig zerlegbare artinsche Ringe sind selbstdual, Arch. Math. 43 (1984), 132-136.

4. C. FAith, Algebra I: rings, modules and categories, 2nd edn (Springer, 1981).

5. J. K. HAACK, Self-duality and serial rings, J. Alg. 59 (1979), 345-363.

6. J. Kraemer, (Self-)duality and the Picard group, Commun. Alg. 16 (1988), 2283-2311.

7. G. O. MichleR, Structure of semi-perfect hereditary Noetherian rings, J. Alg. 13 (1968), 327-344.

8. K. Morita, Duality for modules and its application to the theory of rings with minimum condition, Sci. Rep. Tokyo Kyoiku Daigaku A 6 (1958), 83-142.

9. B. J. MüLLER, Links between maximal ideals in bounded Noetherian prime rings, in Methods in rings theory (ed. F. van Ostaeyen), pp. 347-377 (Reidel, Dordrecht, 1984).

10. B. J. Müller, Morita duality: a survey, in Abelian Groups and Modules, Proc. Conf. Udine (Italy), CISM Courses and Lectures, Volume 287, pp. 395-414 (CISM, Udine, 1984).

11. M. H. UPHAM, Localization and completion of FBN hereditary rings, Commun. Alg. 7 (1979), 1269-1307. 